\title{
Revisiting the Role of Antiarrhythmic Drugs in Prevention of Atrial Fibrillation Recurrence: A Single Center Retrospective Review
}

\author{
Daniel AN Mascarenhas ${ }^{\mathrm{a}}$, Munish Sharma ${ }^{\mathrm{b}, \mathrm{c}}$
}

\begin{abstract}
Background: We conducted a retrospective analysis to revisit the efficacy of four different commonly used antiarrhythmic drugs (AADs) in a single community hospital setting in the U.S. We used cardiac implantable electronic devices (CIEDs) to continuously monitor the patients for maintenance of sinus rhythm. The CIEDs in our study included insertable cardiac monitor (ICM), permanent pacemaker (PPM) and cardiac resynchronization therapy-defibrillator (CRT-D). The aim was to compare efficacy of commonly used AADs for maintenance of sinus rhythm in atrial fibrillation (AF) patients.
\end{abstract}

Methods: We conducted our retrospective study in a real world practice setting. We analyzed electronic medical records of 145 consecutive patients with paroxysmal and persistent AF who were treated with AADs for maintenance of sinus rhythm between the period of April 2014 and February 2018.

Results: Total 34 out of 145 patients (23.45\%) had AF recurrence. The mean duration of first AF recurrence in total patient cohort was $18.01 \pm 12$ months. There was no major difference in efficacy in terms of prevention of first episode of AF recurrence among commonly used class III and class IC AADs.

Conclusions: Higher doses clearly seem to be more effective in preventing the recurrence of AF in class III AADs; sotalol and amiodarone. Use of CIEDs helps to continuously monitor patients for recurrence of AF and detects proarrhythmic effects of AADs.

Keywords: Antiarrhythmic drugs; Atrial fibrillation; Cardiovascular implantable electronic devices

\section{Introduction}

Atrial fibrillation (AF) is the most common cardiac arrhythmia

Manuscript submitted April 13, 2018, accepted April 25, 2018

aDepartment of Cardiology, Drexel University College of Medicine, Easton Hospital, Easton, PA, USA

bepartment of Internal Medicine, Easton Hospital, Easton, PA, USA

${ }^{\mathrm{c} C}$ Corresponding Author: Munish Sharma, Department of Internal Medicine,

Easton Hospital, Easton, PA 18042, USA. Email: munishs1@hotmail.com

doi: https://doi.org/10.14740/cr724w encountered by the clinicians globally. There were estimated 33.5 million individuals with $\mathrm{AF}$ in 2010 according to a review of worldwide population-based studies [1]. Prevalence of AF in the United States was 3.03 million in the year 2005 and this has been projected to increase to 7.56 million by the year 2050 [2]. With the increasing burden of AF globally, its clinical consequences and impact on mortality, morbidity and quality of life per se is a subject of concern. Oftentimes, patients present with symptoms such as palpitations, fatigue, shortness of breath and overall impaired quality of life. Even after adequate control of the ventricular rate, impairment in cardiac performance can lead to reduced exercise tolerance and occasionally to congestive heart failure [3, 4]. AF is associated with around 4-fold increase in risk of stroke, especially in patients not on oral anticoagulation $(\mathrm{OAC})$ and 2-fold increase in death $[3,5]$. To reduce the risk of embolism, control symptoms and improve functional quality, restoration of sinus rhythm is done. However, around $50 \%$ of such patients have recurrent $\mathrm{AF}$ within 6 months duration [3, 6]. Antiarrhythmic drugs (AADs) are used to maintain restoration of sinus rhythm however long-term efficacy in maintaining sinus rhythm and concern about various adverse effects have limited their use among the clinicians [7]. There have been several nonrandomized and randomized trials that were conducted to determine efficacy of AADs but they were focused mainly on a single $\mathrm{AAD}[3,7,8]$. Moreover, studies comparing efficacy of different AADs have not been conducted frequently in the recent years. We conducted a retrospective analysis to revisit the efficacy of four different commonly used AADs in a single community hospital setting in the U.S. We used cardiac implantable electronic devices (CIEDs) to continuously monitor the patients for maintenance of sinus rhythm. The CIEDs in our study included insertable cardiac monitor (ICM), permanent pacemaker (PPM) and cardiac resynchronization therapy-defibrillator (CRT-D).

The objective of our study was to compare efficacy of commonly used AADs for maintenance of sinus rhythm in AF. Determination of improvement in mortality with AADs was beyond the scope of this study.

\section{Methods}

Study setting, patient cohort and inclusion and exclusion criteria

We conducted our retrospective study in a real world practice setting. We analyzed electronic medical records of 145 consecu- 
Table 1. Baseline Clinical Characteristics of the Patients

\begin{tabular}{|c|c|c|c|c|c|}
\hline Baseline characteristics & Total patient cohort & Sotalol group & Amiodarone group & Propafenone group & Flecainide group \\
\hline Total patients & 145 & $66(45.52 \%)$ & $65(44.83 \%)$ & $12(8.28 \%)$ & $2(1.37 \%)$ \\
\hline Sex: female & $74(51.03 \%)$ & $31(21.38 \%)$ & $31(21.38 \%)$ & $11(7.59 \%)$ & $1(0.68 \%)$ \\
\hline $\begin{array}{l}\text { History of coronary } \\
\text { artery disease }\end{array}$ & $89(61.38 \%)$ & $47(32.41 \%)$ & $39(26.90 \%)$ & $3(2.07 \%)$ & 0 \\
\hline $\begin{array}{l}\text { History of moderate to } \\
\text { severe valvular heart disease }\end{array}$ & $24(16.55 \%)$ & $12(8.28 \%)$ & $11(7.59 \%)$ & 0 & $1(0.68 \%)$ \\
\hline Hypertension & $139(95.86 \%)$ & $63(43.45 \%)$ & $62(42.75 \%)$ & $12(8.28 \%)$ & $2(1.38 \%)$ \\
\hline Diabetes mellitus & $26(22.61 \%)$ & $14(12.17 \%)$ & $10(8.70 \%)$ & $2(1.74 \%)$ & 0 \\
\hline $\begin{array}{l}\text { Type of AF(paroxysmal } \\
\text { versus persistent) }\end{array}$ & $\begin{array}{l}\text { Paroxysmal } 134 \\
(92.41 \%) ; \text { Persistent } \\
11(7.59 \%)\end{array}$ & $\begin{array}{l}\text { Paroxysmal } \\
61(42.06 \%) ; \\
\text { Persistent } 5(3.45 \%)\end{array}$ & $\begin{array}{l}\text { Paroxysmal } 59 \\
(40.69 \%) ; \text { Persistent } \\
6(4.14 \%)\end{array}$ & $\begin{array}{l}\text { Paroxysmal } 12 \\
(8.28 \%) ; \text { Persistent } \\
0\end{array}$ & $\begin{array}{l}\text { Paroxysmal } \\
2(1.38 \%) ; \\
\text { Persistent } 0\end{array}$ \\
\hline
\end{tabular}

tive patients with paroxysmal and persistent $\mathrm{AF}$ who were treated with AADs for maintenance of sinus rhythm between the period of April 2014 and February 2018. All patients met the following inclusion criteria: 1) Paroxysmal and persistent AF as defined by 2014 American Heart Association/American College of Cardiology/Heart Rhythm Society guidelines on AF management [9]; 2) Patients who took AADs regularly and followed up with the primary cardiologist at his office regularly; 3) For those with clinically detected index AF event who underwent ICM implantation for continuous rhythm monitoring; 4) For those with pre-existing PPM and CRT-Ds, evidence of AF detected by device. Patients were excluded for the following criteria: 1) Those that had permanent AF; 2) Patients who underwent AF ablation or left atrial appendage closure device placement; 3) Patients who were noncompliant to AAD; 4) Patients who could not tolerate AADs. All the patients were counseled in detail about the benefits and potential risk of AADs. Informed consent was obtained from all the patients before initiation of treatment.

\section{Management protocol applied}

After an episode of clinically detected index AF or device detected AF in patients with pre-existing PPM and CRT-Ds, all patients were started on direct acting oral anticoagulation or warfarin and sinus rhythm was restored with cardioversion after 3 weeks of anticoagulation. Patients were started on an AAD by the end of the third week of index AF event to maintain sinus rhythm. ICM was implanted during the time of initiation of $\mathrm{AAD}$ in patients with first episode of clinically detected AF. Continuous rhythm monitoring was done by means of CIEDs remotely and during office visits. If any patient had more than $6 \mathrm{~h}$ of continuous AF or cumulatively more than $24 \mathrm{~h}$ of AF in 30 consecutive days, their $\mathrm{AAD}$ doses were titrated or a change in AAD itself was made.

\section{Follow-up}

Patients were followed up during the office visits and also dur- ing hospitalizations. Patients were continuously monitored via remote monitoring device for maintenance of sinus rhythm. Regular telephone interviews were carried out to assess patients for symptoms of AF, complications of AF including transient ischemic attack/stroke and adverse events of AAD.

\section{Endpoints}

Primary end point was the length of time to the first recurrence of AF while on AAD whereas the secondary endpoints were adverse effects related to the AAD, thromboembolic events and all cause deaths.

\section{Statistical analysis}

Categorical data were presented as frequencies and percentages and continuous data were displayed as mean \pm standard deviation. We used Student's $t$-tests to compare single variable between two different groups. A P-value of $<0.05$ was considered statistically significant.

\section{Results}

\section{Clinical characteristics}

A total of 145 patients (female: $74,51.03 \%$; mean age $77 \pm$ 6.73 ) met the inclusion criteria for this study. Detail clinical characteristics of these patients are shown in Table 1.

\section{Antiarrhythmic drugs specifications}

Initial choice of AAD was as per the discretion of the treating cardiologist but conventional practice guidelines were followed in choosing AAD. Basic criteria of selection for each of 
Table 2. Antiarrhythmic Drugs Used

\begin{tabular}{|c|c|c|}
\hline Antiarrhythmic drug & Basic criteria of initial choice & Main adverse effects monitored \\
\hline Amiodarone & $\begin{array}{l}\text { Age }>75 \text { years, age }<75 \text { years with renal insufficiency, } \\
\text { reduced LVEF, as an alternative to failed first choice agent }\end{array}$ & $\begin{array}{l}\text { Arrhythmias, LFT derangement, pulmonary toxicity, } \\
\text { skin reaction, thyroid dysfunction, hypotension }\end{array}$ \\
\hline Sotalol & $\begin{array}{l}\text { Age } 65-75 \text { years with normal renal function as first } \\
\text { preference, as an alternative to failed first choice agent }\end{array}$ & $\begin{array}{l}\text { Arrhythmias, bronchospasm, lupus like reaction, } \\
\text { CHF, avoided in aortic stenosis due to LVH }\end{array}$ \\
\hline Propafenone & Age $<65$ years & $\begin{array}{l}\text { Arrhythmias, CHF, myasthenia gravis exacerbation, } \\
\text { agranulocytosis }\end{array}$ \\
\hline
\end{tabular}

LVEF: left ventricular ejection fraction; LFT: liver function test; CHF: congestive heart failure. Cardiac arrhythmias included but not limited to: QT prolongation, torsades, new or exacerbation of ventricular arrhythmias, atrioventricular block and bradycardia.

the AAD used in the study have been summarized in Table 2. A total of 66 patients $(45.52 \%)$ were started on sotalol at the beginning of this study. Out of these patients, total 17 patients had change in their dose of sotalol or change in sotalol itself. In two patients, sotalol was increased from $40 \mathrm{mg}$ twice a day to $80 \mathrm{mg}$ twice a day while in nine patients $80 \mathrm{mg}$ twice a day was increased to $160 \mathrm{mg}$ twice a day to maintain normal sinus rhythm. Three patients were switched from sotalol to propafenone while three of them were switched to amiodarone. Out of 65 patients $(44.83 \%)$ initially started on amiodarone, seven had their dose increased from $100 \mathrm{mg}$ daily to 200 $\mathrm{mg}$ daily, in one patient dose was escalated to $200 \mathrm{mg}$ twice a day, three patients had their amiodarone switched over to sotalol while other two had it changed to propafenone. Out of 12 patients $(8.28 \%)$ on propafenone, one patient's dose was increased from $325 \mathrm{mg}$ twice daily to $425 \mathrm{mg}$ twice a day while the patient had to be changed to sotalol. Among two patients on flecainide $(1.37 \%)$, one patient did well on daily maintenance dose of $100 \mathrm{mg}$ twice a day while other patient had his dose increased to $100 \mathrm{mg}$ three times a day.

\section{Recurrence of first AF with different AADs}

Total 34 out of 145 patients $(23.45 \%)$ had AF recurrence. The mean duration of first AF recurrence in total patient cohort was
$18.01 \pm 12$ months. Seventeen patients on sotalol and 13 on amiodarone had AF recurrence. The failure percentage for sotalol was $25.76 \%$ among the patients started on sotalol while failure for amiodarone was noticed in $20 \%$ patients initially started on amiodarone. There were three cases of AF recurrence in propafenone group ( $25 \%$ recurrence) while one patient had AF recurrence in flecainide group (50\%). Mean duration of first AF recurrence with different AADs and their statistical significance is shown in Tables 3 and 4.

\section{Dosing considerations in AADs}

In sotalol group, 30 patients were started on $80 \mathrm{mg}$ twice daily dose while four patients were started on $40 \mathrm{mg}$ twice daily and 18 patients on 160 twice daily. The difference in average daily dose in sotalol group in patients who had AF recurrence compared to those who did not have AF recurrence was found to be statistically significant. This implied that patients maintained on average daily dose of $207.18 \pm 83.54 \mathrm{mg}$ had no AF recurrence compared to those on $147.69 \pm 30.04$ daily doses. Notably, none of the 18 patients initially started on $160 \mathrm{mg}$ twice daily dose of sotalol had AF recurrence. In amiodarone group, 44 patients were started on 200 daily dose of amiodarone while four were started on $200 \mathrm{mg}$ twice daily and one on $100 \mathrm{mg}$ daily. Patients maintained on average daily dose of

Table 3. Comparatively Showing Number of Patients and Mean Duration of First AF Recurrence

\begin{tabular}{lllllll}
\hline AF recurrence & Sotalol & Amiodarone & Propafenone & Flecainide & $\begin{array}{l}\text { Combined class IC } \\
\text { (propafenone + flecainide) }\end{array}$ & $\begin{array}{l}\text { Combined class III } \\
\text { (amiodarone + sotalol) }\end{array}$ \\
\hline $\begin{array}{l}\text { Number of patients with } \\
\text { recurrence (N) }\end{array}$ & 17 & 13 & 3 & 1 & 4 & 30 \\
$\begin{array}{l}\text { Mean duration of first AF } \\
\text { recurrence } \pm \text { SD (months) }\end{array}$ & $18 \pm 14.85$ & $17.38 \pm 9.90$ & $21.33 \pm 22.81$ & $18.5 \pm 0$ & $20.63 \pm 18.68$ & $17.69 \pm 12.37$ \\
\hline
\end{tabular}

Table 4. Statistical Significance for Primary End Point Among AADs

\begin{tabular}{|c|c|c|c|c|c|c|}
\hline & $\begin{array}{l}\text { Sotalol vs. } \\
\text { amiodarone }\end{array}$ & $\begin{array}{l}\text { Sotalol vs. } \\
\text { propafenone }\end{array}$ & $\begin{array}{l}\text { Sotalol vs. } \\
\text { class IC }\end{array}$ & $\begin{array}{l}\text { Amiodarone vs. } \\
\text { propafenone }\end{array}$ & $\begin{array}{l}\text { Amiodarone } \\
\text { vs. class IC }\end{array}$ & $\begin{array}{l}\text { Class III vs. } \\
\text { class IC }\end{array}$ \\
\hline $\mathrm{P}$-value for duration of first AF recurrence & $0.90(\mathrm{NS})$ & 0.75 (NS) & $0.77(\mathrm{NS})$ & $0.63(\mathrm{NS})$ & $0.64(\mathrm{NS})$ & $0.68(\mathrm{NS})$ \\
\hline
\end{tabular}

NS: statistically not significant. $\mathrm{P}<0.05$ was considered statistically significant. 
Table 5. Different Average Daily Dose of AADs and Recurrence of AF

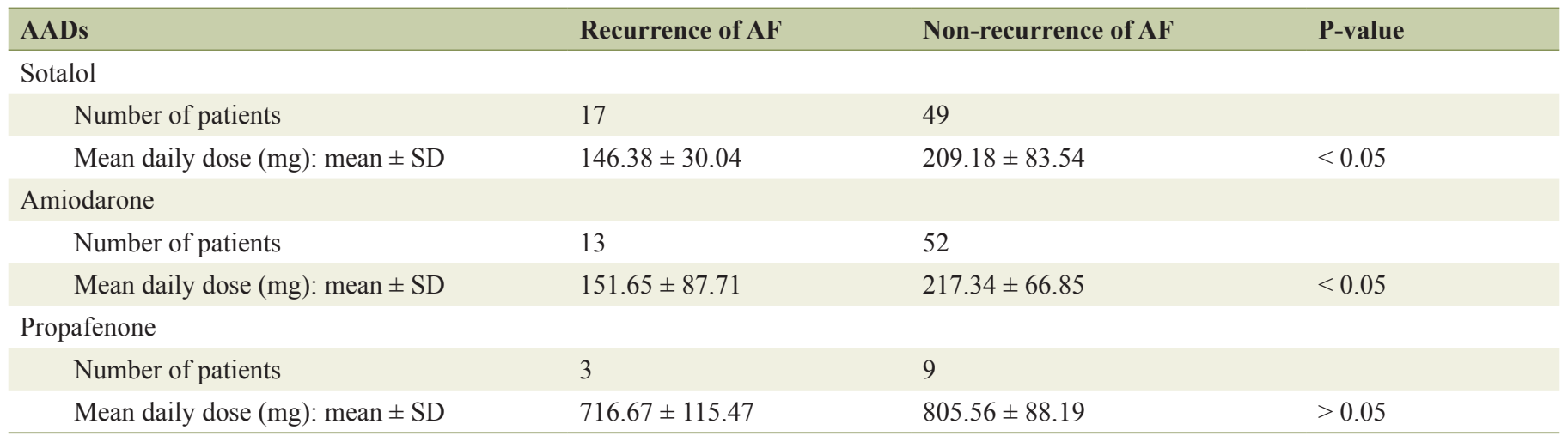

$219.44 \pm 66.85 \mathrm{mg}$ had no AF recurrence compared to those on $153.85 \pm 87.71$. In propafenone group, four patients were started on $325 \mathrm{mg}$ twice daily while the remaining on $425 \mathrm{mg}$ twice daily. The mean dose difference on patients with and without AF recurrence was $88.99 \mathrm{mg}$ per day but it was not statistically significant. This could have been due to small sample size in propafenone group. Similar calculations were not done for flecainide as the total sample size was only two (Table 5).

\section{Secondary end points}

None of the patients had antiarrhythmic drug related adverse effects. All the changes in AAD were prompted by AF recurrence. There were two patients with thromboembolism before the study was started but no patient had evidence of thromboembolic events during this study. During this study period, three patients on sotalol died. Out of these patients, one patient died of exacerbation of chronic respiratory failure (duration of $\mathrm{AAD}=16$ months), one patient died of carcinoma prostate (duration of $\mathrm{AAD}=20$ months) while one patient had subdural hematoma (duration of $\mathrm{AAD}=27$ months). One patient was on amiodarone for 12 months died due to myasthenia-related complications. There were no CIEDs related complications. There were two patients who were lost to follow-up.

\section{CIED specification}

A total of 121 patients had ICM implanted after clinically detected index episode of AF. Reveal /LINQ (Medtronic Inc, MN, USA) ICM were used in 91 patients while BioMonitor 2AF (BIOTRONIK) was used in 30 patients. Twenty-two patients had dual-chamber PPM in device detected first episode of AF. Two patients had CRT-Ds inserted. These devices were upgraded during the course of this study if clinically indicated.

\section{Discussion}

There are two main treatment strategies in AF: rhythm control and rate control. Rhythm control is achieved by restoration and maintenance of normal sinus rhythm with help of AADs or catheter ablation; and rate control is achieved with atrioventricular (AV) nodal blockers. Both rate and rhythm control strategies have not been found to decrease mortality and serious morbidity, such as thromboembolic risk [10, 11]. Rhythm control is preferred mainly in three different settings: 1) In patients with persistent symptoms such as lightheadedness, syncope, angina, dyspnea, palpitation despite adequate rate control; 2) For prevention of tachycardia-mediated cardiomyopathy and 3) Patient's strong preference to stay in normal sinus rhythm irrespective of the nature of AF [12]. American Heart Association/American College of Cardiology/Heart Rhythm Society (ACC/AHA/HRS) (2014) and the European Society of Cardiology (ESC) (2016) both agree that AAD can be used to decrease the frequency and episodes of $\mathrm{AF}$ and improve the quality of life through symptom reduction [9, 13]. Factors precipitating AF, need for anticoagulation and rate control are other strategies in AF management that need to be addressed even in patients maintaining sinus rhythm on AAD. Studies have shown that amiodarone, sotalol, dronedarone, dofetilide, flecainide and propafenone are more effective than placebo for maintenance of normal sinus rhythm and there are few comparative studies between these agents aimed at determining comparative efficacy for maintenance of sinus rhythm [14, 15]. Some studies have associated amiodarone with having greatest efficacy in maintenance of normal sinus rhythm $[14,15]$ while other studies have shown that there is no difference in efficacy in terms of prevention of AF recurrence between amiodarone and sotalol [8]. Thus, our study was designed primarily to revisit the efficacy of all the commonly used AADs in the same clinical setting and determine any difference existing between them. As highlighted in the results section, our study does not reveal any difference between five commonly used AADs; amiodarone, sotalol, dofetilide, flecainide and propafenone. Due to the concern about frequent episodes of proarrhythmia, quinidine, disopyramide and procainamide are no longer recommended for patients with AF for the purpose of maintenance of sinus rhythm [16] and were not used in our patient cohort. We did not have any patient on dronedarone and thus could not make any comments regarding the efficacy of this agent. The starting and maintenance doses of AAD depend on factors such as patient's age, sex, weight, hepatic function and 
renal function. We did find in our study that higher doses of sotalol and amiodarone had fewer recurrence of AF compared to dose on lower doses. This can be considered an interesting and unique finding of our study. This should also encourage other investigators to consider effective dosing with AADs for maintenance of normal sinus rhythm.

Effect on long-term mortality with AAD has always been subject of interest in many studies. A meta-analysis conducted in 2012 included 56 studies (20,771 patients) and compared mortality amongst class IA and class III agents. Quinidine, and disopyramide and sotalol were concluded to be associated with higher all-cause mortality in comparison with amiodarone, dronedarone and dofetilide [17]. While long-term mortality remains to be an important topic in terms of patient safety, it was not within the scope of our single center retrospective study. Proarrhythmia remains to be major concern with use of AAD. Additionally, a drug initially deemed to be safe may have a proarrhythmic effect once patient develops ischemic heart disease or congestive heart failure. There are also concerns about potentially serious drug interactions while on AAD. According to the ACC/AHA/ESC guidelines [18], we monitored patients specifically for widening of QRS to more than $150 \%$ of the baseline QRS duration with type IC drugs. We also make sure in our study that patient's corrected QT interval in sinus rhythm but remained less than $520 \mathrm{~ms}$. During the follow-up with the primary cardiologist, regular monitoring of serum creatinine, serum potassium and serum magnesium were done and changes in the dose or AAD were made accordingly. There was no significant proarrhythmia including ventricular tachycardia, torsades de pointes or ventricular fibrillation in our study cohort. However, larger patient cohort and longer duration of the study could have revealed proarrhythmic adverse effects.

Without an objective and continuous monitoring of cardiac rhythm by implantable devices, it is not easy to accurately determine the efficacy and adverse effects of AAD. Short-term rhythm monitoring with event monitor or Holter monitor for a period ranging from 1 week to 1 month may miss brief intermittent periods of AF or other arrhythmias [19]. Thus, continuous rhythm monitoring to detect episodes of AF recurrence and proarrhythmias/bradycardia potentially related to AAD was another important aspect of our study. Use of CIED (ILR/ PPM/CRT-D) helped us to titrate AAD to achieve sinus rhythm and help to objectively identify any possible proarrhythmic effects with AADs throughout the duration of follow-up. This helped to achieve individualized approach in use of AAD and enhanced patient safety tremendously. To our knowledge, there have not been studies comparing efficacy of AAD where continuous rhythm monitoring by CIED was strictly applied.

\section{Limitations}

This study is a single center retrospective review and the total patient cohort is relatively small. Incorporation of a control group would have made the comparative data on impact of $\mathrm{AAD}$ in prevention of AF recurrence more elaborate. Despite these limitations, this study provides a basis for a larger and adequately powered study.

\section{Conclusions}

The results of our study show that there is no major difference in efficacy in terms of prevention of first episode of AF recurrence among commonly used class III and class IC AADs. These medications should be tailored to the need of the patient and titrated to maintain normal sinus rhythm or achieve low AF burden. Higher doses clearly seem to be more effective in preventing the recurrence of AF in class III AADs; sotalol and amiodarone. Use of CIEDs helps to continuously monitor patients for recurrence of AF and detect proarrhythmic effects of AADs.

\section{Conflict of Interest}

The authors declare no conflict of interest.

\section{References}

1. Lip GYH, Brechin CM, Lane DA. The global burden of atrial fibrillation and stroke: a systematic review of the epidemiology of atrial fibrillation in regions outside North America and Europe. Chest. 2012;142(6):1489-1498.

2. Naccarelli GV, Varker H, Lin J, Schulman KL. Increasing prevalence of atrial fibrillation and flutter in the United States. Am J Cardiol. 2009;104(11):1534-1539.

3. Roy D, Talajic M, Dorian P, Connolly S, Eisenberg MJ, Green M, Kus T, et al. Amiodarone to prevent recurrence of atrial fibrillation. Canadian Trial of Atrial Fibrillation Investigators. N Engl J Med. 2000;342(13):913-920.

4. Morris JJ, Jr., Entman M, North WC, Kong Y, McIntosh $\mathrm{H}$. The changes in cardiac output with reversion of atrial fibrillation to sinus rhythm. Circulation. 1965;31:670678.

5. Risk factors for stroke and efficacy of antithrombotic therapy in atrial fibrillation. Analysis of pooled data from five randomized controlled trials. Arch Intern Med. 1994;154(13):1449-1457.

6. Pritchett EL. Management of atrial fibrillation. N Engl J Med. 1992;326(19):1264-1271.

7. Flaker GC, Blackshear JL, McBride R, Kronmal RA, Halperin JL, Hart RG. Antiarrhythmic drug therapy and cardiac mortality in atrial fibrillation. The Stroke Prevention in Atrial Fibrillation Investigators. J Am Coll Cardiol. 1992;20(3):527-532.

8. Coplen SE, Antman EM, Berlin JA, Hewitt P, Chalmers TC. Efficacy and safety of quinidine therapy for maintenance of sinus rhythm after cardioversion. A meta-analysis of randomized control trials. Circulation. 1990;82(4):1106-1116.

9. January CT, Wann LS, Alpert JS, Calkins H, Cigarroa JE, Cleveland JC, Jr., Conti JB, et al. 2014 AHA/ACC/HRS guideline for the management of patients with atrial fibrillation: executive summary: a report of the American College of Cardiology/American Heart Association Task Force on practice guidelines and the Heart Rhythm Soci- 
ety. Circulation. 2014;130(23):2071-2104.

10. Wyse DG, Waldo AL, DiMarco JP, Domanski MJ, Rosenberg Y, Schron EB, Kellen JC, et al. A comparison of rate control and rhythm control in patients with atrial fibrillation. N Engl J Med. 2002;347(23):1825-1833.

11. Van Gelder IC, Hagens VE, Bosker HA, Kingma JH, Kamp O, Kingma T, Said SA, et al. A comparison of rate control and rhythm control in patients with recurrent persistent atrial fibrillation. N Engl J Med. 2002;347(23):1834-1840.

12. Snow V, Weiss KB, LeFevre M, McNamara R, Bass E, Green LA, Michl K, et al. Management of newly detected atrial fibrillation: a clinical practice guideline from the American Academy of Family Physicians and the American College of Physicians. Ann Intern Med. 2003;139(12):1009-1017.

13. Kirchhof P, Benussi S, Kotecha D, Ahlsson A, Atar D, Casadei B, Castella M, et al. 2016 ESC Guidelines for the management of atrial fibrillation developed in collaboration with EACTS. Eur Heart J. 2016;37(38):2893-2962.

14. McNamara RL, Tamariz LJ, Segal JB, Bass EB. Management of atrial fibrillation: review of the evidence for the role of pharmacologic therapy, electrical cardioversion, and echocardiography. Ann Intern Med. 2003;139(12):1018-1033.
15. Lafuente-Lafuente C, Mouly S, Longas-Tejero MA, Mahe I, Bergmann JF. Antiarrhythmic drugs for maintaining sinus rhythm after cardioversion of atrial fibrillation: a systematic review of randomized controlled trials. Arch Intern Med. 2006;166(7):719-728.

16. Singh BN, Singh SN, Reda DJ, Tang XC, Lopez B, Harris $\mathrm{CL}$, Fletcher RD, et al. Amiodarone versus sotalol for atrial fibrillation. N Engl J Med. 2005;352(18):18611872.

17. Lafuente-Lafuente C, Longas-Tejero MA, Bergmann JF, Belmin J. Antiarrhythmics for maintaining sinus rhythm after cardioversion of atrial fibrillation. Cochrane Database Syst Rev. 2012;5:CD005049.

18. Wann LS, Curtis AB, January CT, Ellenbogen KA, Lowe JE, Estes NA, 3rd, Page RL, et al. 2011 ACCF/AHA/HRS focused update on the management of patients with atrial fibrillation (Updating the 2006 Guideline): a report of the American College of Cardiology Foundation/American Heart Association Task Force on Practice Guidelines. J Am Coll Cardiol. 2011;57(2):223-242.

19. Mascarenhas DA, Farooq MU, Ziegler PD, Kantharia BK. Role of insertable cardiac monitors in anticoagulation therapy in patients with atrial fibrillation at high risk of bleeding. Europace. 2016;18(6):799-806. 